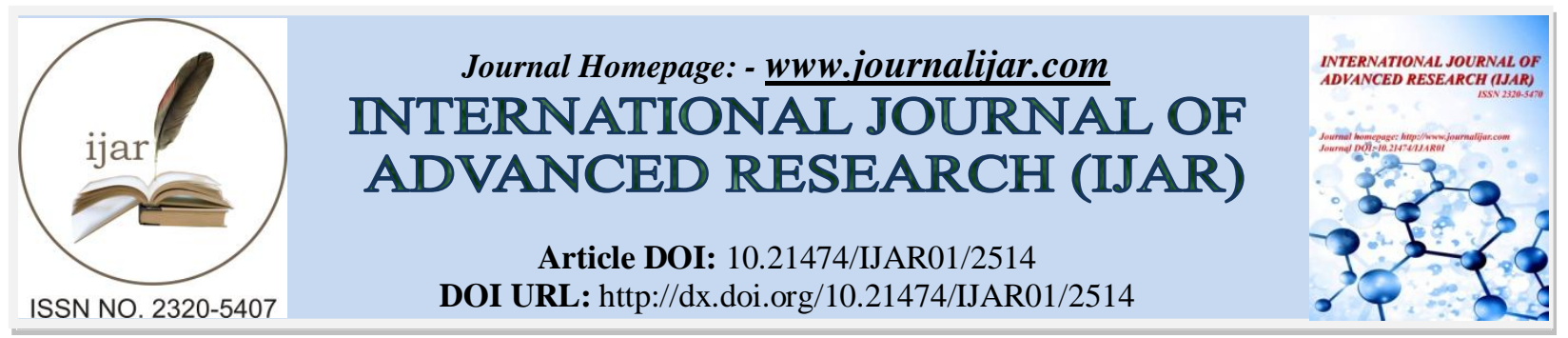

RESEARCH ARTICLE

\title{
HIGH CDX2 GENE EXPRESSION PREDICTS INFERIOR PROGNOSIS IN ACUTE MYELOID LEUKEMIA PATIENTS WITH NORMAL CYTOGENETICES.
}

\section{Shereen Mohamed El-Hoseiny MD', Hanan Ali Taha MD², Nilly Helmy Mohamed MD² and Sara Ahmed Sayed ${ }^{2}$.}

1. Department of Clinical and Chemical Pathology Beni Suef Teaching Hospital, Faculty of Medicine, BENISUEF UNIVERSITY Egypt

2. Department of Internal Medicine, Beni Suef Teaching Hospital, Faculty of Medicine, BENI-SUEF UNIVERSITY, Egypt.

\section{Manuscript Info}

Manuscript History

Received: 25 October 2016

Final Accepted: 23 November 2016

Published: December 2016

Key words:-

CDX2, CN-AML, RTQ-PCR.

\section{Abstract}

Background: The caudal-type homebox gene CDX2 plays an important role not only in normal hematopoiesis but also in leukemogenesis. It is one of the most frequent aberrantly expressed proto-oncogenes in human leukemias.

Patients and Methods: We studied the expression level of CDX2 mRNA in 66 newly diagnosed cytogenetically normal acute myeloid leukemia (CN-AML) patients and 20 healthy controls by Real-Time Quantitative Reverse-Transcriptase Polymerase Chain Reaction (RTQ-PCR) to determine gene relation to patients clinical and laboratory findings at diagnosis and to determine its impact on patients' outcome.

Results: CDX2 expression rates and expression levels were significantly higher in AML patients than in controls $(\mathbf{p}<\mathbf{0 . 0 0 1})$. CDX2 high expressors were significantly older $(\mathbf{p}=\mathbf{0 . 0 0 6})$, had higher TLC (p=0.001), lower platelets count $(\mathbf{p = 0 . 0 2 4 )}$ and had hepatosplenomegaly $(\mathbf{p}<\mathbf{0 . 0 0 1})$ and lymph node enlargement $(\mathbf{p = 0 . 0 0 4})$ more than CDX $2^{\text {low }}$ expressors. CDX2 ${ }^{\text {high }}$ expressors was associated with worse prognosis; worse CR rates, shorter OS,DFS and EFS than CDX2 low expressors. The difference were highly statistically significant $(\mathbf{p}<\mathbf{0 . 0 0 1})$ in all.

Conclusion: $\mathrm{CDX} 2$ is highly expressed in $\mathrm{CN}$-AML patients. It is associated with high tumor burden and unfavorable prognosis. Therefore, it could be added to the prognostic markers in CN-AML patients.

\section{Introduction:-}

Cytogenetic is considered the most important prognostic factor in patients with AML, however, 40-50\% of AML patients are cytogenetically normal (CN-AML) (Mrozek et al., 2004). These patients usually fall into intermediaterisk cytogenetics, but show significantly heterogeneous outcomes (Dohner et al., 2010). Leukemic blasts of CNAML patients don't have any detectable chromosomal abnormalities by conventional cytogenetic techniques, but they may hide mutations, epigenetic changes and aberrantly expressed proteins or micro RNAs, which are used for 
risk classification and prognostic evaluation of AML patients (Deneberg et al., 2011). These include mutations of CEBPA (Li et al., 2015), NPMI (Dohner et al., 2005), which are associated with favorable prognosis, while mutations of FLT3-ITD (Santos et al., 2011), WT1 (Lyu et al., 2014), BAALC (Schwind et al., 2010) and ERG (Schwind et al., 2010) are associated with unfavorable prognosis. CN-AML has different response to chemotherapy and different prognosis, so identification of new biomarkers for their diagnosis and prognosis is an urgent need (Shi et al., 2016).

Two classes of Homebox genes are known; Class I: clustered genes (Hox) genes which contain 39 members; Class II: divergent homeobox genes which include smaller families as PAX and Para Hox (CDX) genes (Argiropoulos et al., 2007). The caudal -type homebox gene CDX2 belongs to the ParaHox A cluster which exists on chromosome 13q12 (Garcia-Fernandez 2005). CDX2 codes for a transcription factor that regulates embryonic organogenesis and early hematopoietic development in vertebrates (Abramovich \& Humphries 2005). CDX2 and other CDX family acts through regulation of Hox gene expression, CDX proteins can also act directly on Hox regulatory elements (Chawengsaksophak et al., 2004).

Hox genes are important for hematopoietic development and maintenance; they are expressed in hematopoietic precursors (Pineault et al., 2002). Aberrant expression of CDX2 in hematopoietic compartment may be associated with altered expression of Hox genes (Scholl et al 2007).

CDX2 genes are able to transform hematopoietic progenitors into leukemic stem cells either by upstream regulation of Hox genes or by expression of several Hox genes at the same time. CDX2 was found to be one of the most frequent aberrantly expressed proto-oncogenes in human leukemia especially in AML (Rawat et al., 2012). This may be due to dysregulation of Hox genes (Scholl et al 2007).

From this context, this case-control study was done to determine the influence of CDX2 gene in CN-AML patients and to study gene relation to patients clinical and laboratory data at diagnosis and its impact on patients' response to therapy.

\section{Patients and Methods:-}

The present study was carried on 66 newly diagnosed cytogenetically normal acute myeloid leukemia (CN-AML) patients, together with 20 healthy unrelated age and sex matched controls. Patients and controls were recruited from Beni Suef University Hospital and National Cancer Institute (NCI), Cairo University after taking their informed contest. Ethical considerations were done according to the Declaration of Helsinki of Bioethics (2008).

AML was diagnosed according to the morphological findings of BM aspirate stained smears; its cytochemical stains and immunophenotyping criteria's. Cytogenetic analysis of AML BM samples was done using the conventional cytogenetic analysis; in which at least 20 metaphase cells were analyzed and the karyotype in it was normal in order to consider it of normal cytogenetics (CN).AML patients were classified according to the French-Americian-British criteria (FAB) classifications. Patients' characters were shown in Table (1).

\section{Treatment of AML patients:-}

Patients were treated according to the AML standard protocol of the NCI, Cairo University. Patients received induction chemotherapy (3-7 protocol) which consists of 3 days of Daunorubicin $45 \mathrm{mg} / \mathrm{m}^{2}$ at $15-30$ minute infusion, combined with arabinosylcytosine (Ara-C) $100 \mathrm{mg} / \mathrm{m}^{2}$ as a 24 hour daily infusion for 7 days. This protocol was repeated if the patient didn't achieve complete remission (CR), and patients were shifted to higher dose chemotherapy if no or minimal response was achieved (Tallman 2005).

\section{Assessment of patients' response to therapy:-}

After completion of 28 days of induction therapy; remission status was assessed. Complete Remission (CR) was defined as granulocytic count of $1.5 \times 10^{9} / \mathrm{L}$ at least, platelets count of $100 \times 10^{9} / \mathrm{L}$ at least, no PB blasts, BM cellularity of $20 \%$ at least with maturation of all cell lines and less than $5 \%$ blasts with no extramedullary leukemia. Relapse was defined as reappearance of PB blasts, more than 5\% blasts in BM, or reappearance of extramedullary manifestations after CR was achieved. Overall Survival (OS) was measured from the date of diagnosis till the date of death (regardless of the cause), censoring for patients alive at last follow up. Disease Free Survival (DFS) was defined only for patients who achieved CR; it was measured from date of CR till date of relapse or death regardless of the cause censoring for patients alive at last follow up. Event Free Survival (EFS) was defined for patients who 
achieved CR as the time from the date of diagnosis till relapse or death regardless of the cause, censoring for those alive at last follow up. For patients who didn't achieve CR but expired within 2 months of the on-study date, EFS was defined as the time from study date until death regardless of the cause (Kühnl et al., 2011).

\section{Methods:-}

RNA isolation and Real -Time Quantitative Reverse Transcriptase-Polymerase Chain Reaction (RT-PCR): Mononuclear cells (MNCs) were isolated from peripheral blood (PB) or BM aspirate by ficoll density gradient centrifugation at diagnosis before starting treatment. Follow up of patients was carried out for 2 years. Total RNA was extracted from MNCs using a QIA amp® RNA Blood Mini Kits (Catalog No. 52304), (Qiagen, Germany) according to the manufacturer's instructions. Complementary DNA (cDNA) was synthesized using (dt) 15-mer primer by Superscript III Reverse Transcriptase using High-Capacity cDNA Archieve Kit, Applied Biosystems (Part No. 4322171) and stored at $-20^{\circ} \mathrm{C}$ till use.

The caudal -type homeobox gene (CDX2) mRNA gene expression level and its housekeeping gene (endogenous control) Glyceraldehyde -3-Phosphate Dehydrogenase (GAPDH) were assayed by the TaqMan quantitative RTPCR using an ABI PRISM 7000 Sequence Detector System (Applied Biosystems, Foster City, CA). The quantitative RT-PCR amplification was performed using the pre-developed Assays-on - demand Gene Expression Set for CDX2 (Assay ID: Hs01078080_m1, Catalo No. 4322171, Applied Biosystems), TagMan GAPDH control reagents (Human Part No.402869, Applied Biosystems) and TagMan Universal PCR Master Mix (Catalog No. 4440043, Applied Biosystems).

RTQ-PCR assays were performed for CDX2 and GAPDH in the same tube in a final reaction volume of $25 \mathrm{ul}$ containing $5 \mathrm{ul} \mathrm{cDNA}$. The reaction protocol used involved heating for $2 \mathrm{~min}$ at $50^{\circ} \mathrm{C}$ and $10 \mathrm{~min}$ at $95^{\circ} \mathrm{C}$, followed by $40 \mathrm{PCR}$ cycles of amplification $\left(15 \mathrm{sec}\right.$ at $95^{\circ} \mathrm{C}$ and $1 \mathrm{~min}$ at $\left.60^{\circ} \mathrm{C}\right)$. Analysis was performed using ABI PRISM 7000 Sequence Detection Software (Applied Biosystems) and Applied Biosystem Step One ${ }^{\text {TM }}$ Instrument (USA).

CDX2 gene levels were expressed in the form of cycle threshold (CT) then normalized copy number (relative quantitation) was calculated using the $\Delta \Delta \mathrm{CT}$ equation: $\Delta \Delta \mathrm{CT}=\Delta \mathrm{CT}$ of case- $\Delta \mathrm{CT}$ of control then Relative Quantification $(\mathrm{RQ})=2^{-\Delta \Delta \mathrm{CT}}$. A negative control without template was included in each experiment (Fig 1).

AML patients was classified according to CDX2 median expression level (700) into CDX2 ${ }^{\text {High }}$ expressors if they had expression level above median and CDx $2{ }^{\text {Low }}$ expressors if they had expression level below median expression level.

\section{Statistical Methods:-}

Data was analyzed using IBM SPSS advanced statistics version 22 (SPSS Inc., Chicago, IL). Numerical data were expressed as mean and standard deviation or median and range. Qualitative data were expressed as frequency and percentage. Chi-square test was used to examine the relationship between qualitative variables. Comparison between two quantitative variables that are not normally distributed was done using Mann-Whitney test (non- parametric ttest). While comparison between 3 groups was done using Kruskal-Wallis test (non parametric Anova). Correlation between numerical variables was done using Spearman-rho method. For univariate survival analysis; Kaplan-Meier method was done while comparison between two survival curves were done using log-rank test and Cox- regression analysis for numerical variables. For significant factors affecting survival on univariate analysis; Multivariate analysis was done using Cox-regression method. For risk estimation; Hazard ratio (HR) with its 95\% confidence interval (CI) were used for risk estimation. All tests were two- tailed and a p-value $<0.05$ was considered significant.

\section{Results:-}

The current study was conducted on $66 \mathrm{CN}-\mathrm{AML}$ patients and 20 healthy unrelated controls.

\section{CDX2 Expression:-}

CDX2 mRNA was expressed in all 66/66 AML patients (100\%) while it was expressed in only 6/20 (30\%) of controls. CDX2 mean expression level was 19272.9 75809.3 (range: 5.8-578779.5) in AML patients, while it was 3.0 \pm 1.1 (range: 1.3-4.7) in controls. Both the expression rate and expression levels of CDX2 mRNA were significantly higher in AML patients than in controls $(\mathbf{p}<\mathbf{0 . 0 0 1})$. 
Table (1) shows clinical and laboratory data of the 66 AML patients.

Follow up of all AML patients are represented in Table (2).

Impact of CDX2 Gene on Clinical and Laboratory Data of AML Patients (High versus Low CDX2 expression) (Table 3):-

Patients were classified according to CDX2 median expression level (700.0) into 32 (48.5\%) patients with high CDX2 (above median expression level $>700)$ and $34(51.5 \%)$ patients with low CDX2 ( $\leq 700)$.

There was a statistically significant difference between CDX $2^{\text {high }}$ and CDX2 ${ }^{\text {low }}$ expressors as regards age (p=0.006), TLC (p=0.001), platelets count $(\mathbf{p}=\mathbf{0 . 0 2 4})$, hepatosplenomegaly $(\mathrm{HSM})(\mathbf{p}<\mathbf{0 . 0 0 1})$, LNs enlargement $(\mathbf{p}=\mathbf{0 . 0 0 4})$ and

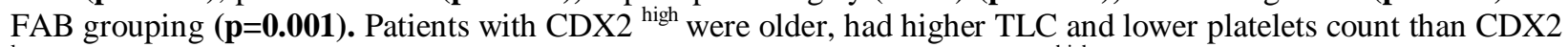
low expressors. HSM and LN enlargement were more encountered in CDX2 high expressors. As for FAB grouping: M0,M1 and M2 were more presented in CDX2 ${ }^{\text {low }}$ expressors while M4, M5 and M6 were more in CDX2 high expressors.

There was no statistically significant difference between CDX2 $2^{\text {high }}$ and CDX2 low expressors regarding gender, hemoglobin level or BM blast percentage $(\mathrm{p}>0.05)$.

CDX2 ${ }^{\text {high }}$ expression associates with bad prognosis, shorter OS, DFS and EFS (Table 4):

CDX2 high expressors patients associate with worse prognosis than CDX2 low as all $20(100 \%)$ patients who didn't achieve complete remission $(\mathbf{C R})$ were in CDX2 ${ }^{\text {high }}$ expressors. CR was more encountered in CDX2 ${ }^{\text {low }}$ expressors (73.9\%) compared to $(26.1 \%)$ in CDX2 high expressors, the difference was highly statistically significant (p<0.001), odds ratio was 3.833(95\% CI: 2.357-6.235).

CDX2 ${ }^{\text {high }}$ expressors were also associated with shorter $\mathbf{O S}$ as cumulative survival at 24 months was $37.1 \%$ in CDX2 high compared to $96.2 \%$ in CDX2 low expressors. The difference was highly statistically significant $(\mathbf{p}<\mathbf{0 . 0 0 1})$, hazards ratio (HR) of death was 15.608(95\% CI: 4.089-59.576) (Fig 2). DFS were shorter in CDX2 high than CDX2

low expressors, cumulative survival at 24 months was $8.3 \%$ in CDX2 ${ }^{\text {high }}$ compared to $89.4 \%$ in CDX2 ${ }^{\text {low }}$ expressors. The difference was highly statistically significant $(\mathbf{p}<\mathbf{0 . 0 0 1})$, HR of relapse and/or death was 21.874 (95\% CI: 7.315-65.410) (Fig 3). Also EFS was shorter in CDX2 high than CDX2 low expressors, cumulative survival at 24months was only $3.1 \%$ in CDX2 high compared to $89.4 \%$ in CDX2 low expressors. The difference was highly statistically significant $(\mathbf{p}<\mathbf{0 . 0 0 1})$ and HR of not achieving CR, relapse and/or death was 30.079(95\% CI: 10.91882.872) (Fig 4).

Multivariate Analysis shows that CDX2 ${ }^{\text {high }}$ expression level associates with worse OS, DFS, EFS (Table 5): On multivariate analysis using Cox regression method of the significant variables on univariate analysis after adjusting for the impact of other known risk factors, the independent factors that influence OS were CDX2 with HR $=21.439$ (95\% CI: 4.765-96.67) and TLC with HR=1.017 (95\% CI: 1.008-1.026). The independent factors that influence DFS were CDX2 with HR = 17.644 (95\% CI: 5.501-56.588) and organomegaly with HR=4.886 (95\% CI: 1.447-16.499). While in EFS, the independent factors affecting it was CDX2 with HR=29.034 (95\%CI: 10.35981.372) and TLC with $\mathrm{HR}=1.005$ (95\%CI: 1.001-1.009). As to say that CDX2 expression level was an independent prognostic factor affecting significantly OS, DFS and EFS. 
Table 1:- Clinical and Laboratory Data of the 66 AML patients.

\begin{tabular}{|c|c|}
\hline Characteristic & Value \\
\hline $\begin{array}{l}\text { CDX2 expression Level: } \\
\text { Median }\end{array}$ & $\begin{array}{l}19272.9 \pm 75809.3 \\
(5.8-578779.5) * \\
662.6\end{array}$ \\
\hline $\begin{array}{l}\text { Gender: No (\%) } \\
\text { Male } \\
\text { Female }\end{array}$ & $\begin{array}{l}31(47.0 \%) \\
35(53.0 \%)\end{array}$ \\
\hline $\begin{array}{l}\text { Age at diagnosis: (years) } \\
\text { Median }\end{array}$ & $\begin{array}{l}47.2 \pm 13.1(20-72) \\
48.5\end{array}$ \\
\hline $\begin{array}{l}\text { Total leucocytic count } \times 10^{9} / \mathrm{L} \\
\text { Median }\end{array}$ & $\begin{array}{l}51.4 \pm 65.8(1.8-378.0) \\
28.7\end{array}$ \\
\hline $\begin{array}{l}\text { Hemoglobin gm/dl } \\
\text { Median }\end{array}$ & $\begin{array}{l}7.6 \pm 2.1(3.9-12.0) \\
7.5\end{array}$ \\
\hline $\begin{array}{l}\text { Platelets } \mathbf{x 1 0}{ }^{9} / \mathbf{L} \\
\text { Median }\end{array}$ & $\begin{array}{l}101.4 \pm 93.7(9-330) \\
60.8\end{array}$ \\
\hline $\begin{array}{l}\text { Bone Marrow blasts } \\
\text { Median }\end{array}$ & $\begin{array}{l}64.2 \pm 20.8(23-100)^{*} \\
64.5\end{array}$ \\
\hline Hepatosplenomegaly (\%) & $15 / 66$ cases $(22.7 \%)$ \\
\hline LN enlargement $(\%)$ & $7 / 66$ cases $(10.6 \%)$ \\
\hline $\begin{array}{l}\text { FAB classification: } \\
\text { M0: No }(\%) \\
\text { M1: No }(\%) \\
\text { M2: No }(\%) \\
\text { M4: No }(\%) \\
\text { M5: No }(\%) \\
\text { M6: No }(\%)\end{array}$ & $\begin{array}{l}6(9.1 \%) \\
20(30.3 \%) \\
22(33.3 \%) \\
9(13.6 \%) \\
6(9.1 \%) \\
3(4.5 \%) \\
\end{array}$ \\
\hline
\end{tabular}

*Mean \pm SD (range) FAB classification: French American British classification

Table 2:- Follow up of the 66 AML patients.

\begin{tabular}{|l|l|}
\hline Characteristic & Value \\
\hline Complete Remission (CR): No (\%) & $46(69.7 \%)$ \\
No CR: No (\%) & $20(30.3 \%)$ \\
\hline Overall Survival (OS): & $48 / 66(72.7 \%)$ \\
\hline Alive: No (\%) & $18 / 66(27.3 \%)$ \\
Dead: No (\%) & $\mathbf{7 2 . 8 \%}$ \\
Cumulative survival at 24 months: \% & $29 / 46(63.0 \%)$ \\
\hline Disease Free Survival (DFS): of the 46 CR patients: & $17 / 46(37.0 \%)$ \\
\hline Alive Free (No Relapse or Death): No (\%) & $\mathbf{6 8 . 1 \%}$ \\
Relapse and/or Death: No (\%) & \\
Cumulative survival at 24 months: \% & \\
\hline Event Free Survival (EFS): & $\mathbf{4 7 . 4 \%}$ \\
\hline Alive Free (CR): No (\%) & \\
Alive Diseased (Relapse or No CR) /or Death: No (\%) & \\
Cumulative survival at 24 months: \% & \\
\hline
\end{tabular}

$*$ Mean \pm SD (range) 
Table 3:- Impact of CDX2 Gene on Clinical and Laboratory Data of the 66 AML Patients (High versus Low CDX2 expression).

\begin{tabular}{|c|c|c|c|}
\hline Characteristic & $\begin{array}{l}\text { High } C D X 2 \\
>700\end{array}$ & $\begin{array}{l}\text { Low } C D X 2 \\
\leq 700\end{array}$ & P value \\
\hline Number (\%) & $32(48.5 \%)$ & $34(51.5 \%)$ & \\
\hline$C D X 2$ expression & $\begin{array}{l}39470.9 \pm 105984.7(765.6-578779.5) \\
*\end{array}$ & $263.0 \pm 205.3(5.8-670.9)$ & \\
\hline Median & 4178.2 & 239.0 & $<0.001$ \\
\hline $\begin{array}{l}\text { Gender: No (\%) } \\
\text { Male } \\
\text { Female }\end{array}$ & $\begin{array}{l}13(41.9 \%) \\
19(54.3 \%)\end{array}$ & $\begin{array}{l}18(58.1 \%) \\
16(45.7 \%)\end{array}$ & 0.316 \\
\hline $\begin{array}{l}\text { Age(years) } \\
\text { Median }\end{array}$ & $\begin{array}{l}51.7 \pm 12.6(22-72) \\
54.0\end{array}$ & $\begin{array}{l}43.0 \pm 12.3(20-65) \\
44.0\end{array}$ & 0.006 \\
\hline $\begin{array}{l}\text { Total leucocytic count } \times 10^{9} / \mathrm{L} \\
\text { Median }\end{array}$ & $\begin{array}{l}77.9 \pm 83.8(2.2-378.0) \\
54.7\end{array}$ & $\begin{array}{l}26.4 \pm 24.4(1.8-119.0) \\
19.7\end{array}$ & 0.001 \\
\hline $\begin{array}{l}\text { Hemoglobin gm/dl } \\
\text { Median }\end{array}$ & $\begin{array}{l}7.5 \pm 2.1(3.9-11) \\
7.4\end{array}$ & $\begin{array}{l}7.7 \pm 2.1(3.9-12) \\
7.6\end{array}$ & 0.594 \\
\hline $\begin{array}{l}\text { Platelets x } 10^{9} / \mathrm{L} \\
\text { Median }\end{array}$ & $\begin{array}{l}72.0 \pm 70.6(9-254) \\
42.5\end{array}$ & $\begin{array}{l}129.2 \pm 104.8(10-330) \\
90.9\end{array}$ & 0.024 \\
\hline $\begin{array}{l}\text { Bone Marrow blasts } \\
\text { Median }\end{array}$ & $\begin{array}{l}60.9 \pm 20.7(29-95) \\
56.5\end{array}$ & $\begin{array}{l}67.2 \pm 20.8(23-100) \\
72.0\end{array}$ & 0.195 \\
\hline $\begin{array}{l}\text { Hepatosplenomegaly }(\%) \\
15 \text { cases }\end{array}$ & $14(93.3 \%)$ & $1(6.7 \%)$ & $<0.001$ \\
\hline $\begin{array}{l}\text { LN enlargement (\%) } \\
7 \text { cases }\end{array}$ & $7(100 \%)$ & $0(0 \%)$ & 0.004 \\
\hline $\begin{array}{l}\text { FAB classification: } \\
\text { M0: No }(\%) 6 \text { cases } \\
\text { M1: No }(\%) 20 \text { cases } \\
\text { M2: No }(\%) 22 \text { cases } \\
\text { M4: No }(\%) 9 \text { cases } \\
\text { M5: No (\%) } 6 \text { cases } \\
\text { M6: No }(\%) 3 \text { cases }\end{array}$ & $\begin{array}{l}4(66.7 \%) \\
6(30.0 \%) \\
7(31.8 \%) \\
6(66.7 \%) \\
6(100 \%) \\
3(100 \%) \\
\end{array}$ & $\begin{array}{l}2(33.3 \%) \\
14(70.0 \%) \\
15(68.2 \%) \\
3(33.3 \%) \\
0(0 \%) \\
0(0 \%) \\
\end{array}$ & \\
\hline $\begin{array}{l}\text { FAB grouping: } \\
\text { M0+M1+M2: : No }(\%) 48 \text { cases } \\
\text { M4+M5+M6: : No }(\%) 18 \text { cases }\end{array}$ & $\begin{array}{l}17(35.4 \%) \\
15(83.3 \%)\end{array}$ & $\begin{array}{l}31(64.6 \%) \\
3(16.7 \%)\end{array}$ & 0.001 \\
\hline
\end{tabular}

$*$ Mean \pm SD (range)

FAB classification: French American British classification

Bold values are statistically significant

P<0.05 Sig; $\quad$ P $<0.001$ HS;

P>0.05 NS.

Sig significant, HS highly significant, NS not significant 
Table 4:- Impact of CDX2 Gene Expression on Follow up of AML patients (High versus Low CDX2).

\begin{tabular}{|c|c|c|c|c|}
\hline Characteristic & $\begin{array}{l}\text { High } C D X 2 \\
32 \text { cases } \\
(48.5 \%)\end{array}$ & $\begin{array}{l}\text { Low } C D X 2 \\
34 \quad \text { cases } \\
(51.5 \%)\end{array}$ & $P$ value & Odds Ratio (95\% CI) \\
\hline $\begin{array}{l}\text { Complete Remission (CR): } 46 \text { cases } \\
\text { No }(\%) \\
\text { No Complete Remission: } 20 \text { cases } \\
\text { No }(\%)\end{array}$ & $\begin{array}{l}12(26.1 \%) \\
20(100 \%)\end{array}$ & $\begin{array}{l}34(73.9 \%) \\
0(0 \%)\end{array}$ & $<0.001$ & $3.833(2.357-6.235)$ \\
\hline Characteristic & $\begin{array}{l}\text { High } C D X 2 \\
32 \text { cases } \\
(48.5 \%) \\
\end{array}$ & $\begin{array}{l}\text { Low } C D X 2 \\
34 \quad \text { cases } \\
(51.5 \%)\end{array}$ & $P$ value & Hazard Ratio (95\% CI) \\
\hline $\begin{array}{l}\text { Overall Survival }(\mathrm{OS}) \\
\text { Alive: }(48 \text { cases)No }(\%) \\
\text { Dead: }(18 \text { cases }) \text { No }(\%)\end{array}$ & $\begin{array}{l}17(35.5 \%) \\
15(83.3 \%)\end{array}$ & $\begin{array}{l}31(64.5 \%) \\
3(16.7 \%)\end{array}$ & \multirow[t]{2}{*}{$<0.001$} & \multirow[t]{2}{*}{$15.608(4.089-59.576)$} \\
\hline $\begin{array}{l}\text { OS: Cumulative survival at } 24 \\
\text { months: } \%\end{array}$ & $37.1 \%$ & $96.2 \%$ & & \\
\hline $\begin{array}{l}\text { Disease Free Survival (DFS): of the } \\
46 \text { CR patients } \\
\text { Alive Free: } 29 \text { cases: No }(\%) \\
\text { Alive Diseased (Relapsed) or Death: } \\
17 \text { cases: No }(\%)\end{array}$ & $\begin{array}{l}0(0.0 \%) \\
12(70.5 \%)\end{array}$ & $\begin{array}{l}29(100 \%) \\
5(29.5 \%)\end{array}$ & \multirow[t]{2}{*}{$<0.001$} & \multirow[t]{2}{*}{21.874 (7.315-65.410) } \\
\hline $\begin{array}{l}\text { DFS: Cumulative survival at } 24 \\
\text { months } \%\end{array}$ & $8.3 \%$ & $89.4 \%$ & & \\
\hline $\begin{array}{l}\text { Event Free Survival (EFS): } \\
\text { Alive Free }(\text { CR): } 29 \text { cases No }(\%) \\
\text { Alive Diseased (Relapse or No CR) } \\
\text { /or Death: } 37 \text { cases No }(\%)\end{array}$ & $\begin{array}{l}0(0.0 \%) \\
32(86.5 \%)\end{array}$ & $\begin{array}{l}29(100 \%) \\
5(13.5 \%)\end{array}$ & \multirow[b]{2}{*}{$<0.001$} & \multirow[b]{2}{*}{$30.079(10.918-82.872)$} \\
\hline $\begin{array}{l}\text { EFS: Cumulative survival at } 24 \\
\text { months: } \%\end{array}$ & $3.1 \%$ & $89.4 \%$ & & \\
\hline
\end{tabular}

CI: Confidence interval

Bold values are statistically significant

P<0.05 Sig; $\quad$ P $<0.001$ HS;

P>0.05 NS.

Sig significant, HS highly significant, NS not significant

Table 5:- Multivariate Analysis by Cox regression showing only the independent factors that influence the OS, DFS and EFS in the 66 AML patients.

\begin{tabular}{|c|c|c|}
\hline Parameters & P-value & Hazard Ratio (95\% CI) \\
\hline $\begin{array}{l}\text { Overall Survival }(\mathrm{OS}): \\
\text { CDX2 Gene Expression Levels: } \\
\text { Total leucocytic count: }\end{array}$ & $\begin{array}{l}<0.001 \\
<0.001 \\
\end{array}$ & $\begin{array}{l}21.439(4.765-96.467) \\
1.017(1.008-1.026)\end{array}$ \\
\hline $\begin{array}{l}\text { Disease Free Survival (DFS): } \\
\text { CDX2 Gene Expression Levels: } \\
\text { Organomegaly (HSM, LNs enlargement): }\end{array}$ & $\begin{array}{l}<0.001 \\
0.011\end{array}$ & $\begin{array}{l}17.644(5.501-56.588) \\
4.886(1.447-16.499)\end{array}$ \\
\hline $\begin{array}{l}\text { Event Free Survival (EFS): } \\
\text { CDX2 Gene Expression Levels: } \\
\text { Total leucocytic count: }\end{array}$ & $\begin{array}{l}<0.001 \\
0.011\end{array}$ & $\begin{array}{l}29.034(10.359-81.372) \\
1.005(1.001-1.009)\end{array}$ \\
\hline
\end{tabular}

CI: Confidence interval

Bold values are statistically significant

P $<0.05$ Sig; $\quad$ P $<0.001$ HS;

Sig significant, HS highly significant, NS not significant

P>0.05 NS. 


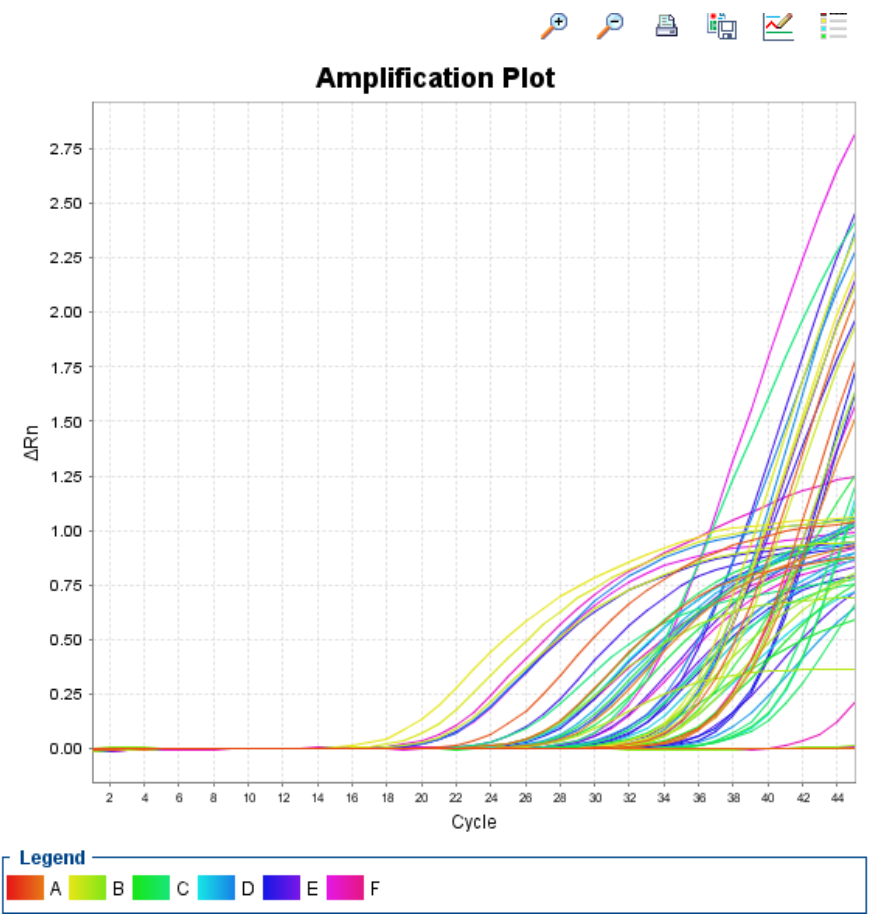

Fig 1:- CDX2 Gene and GAPDH expressions curves in AML cases and controls by Real-time Relative Quantitative PCR (RT-PCR).

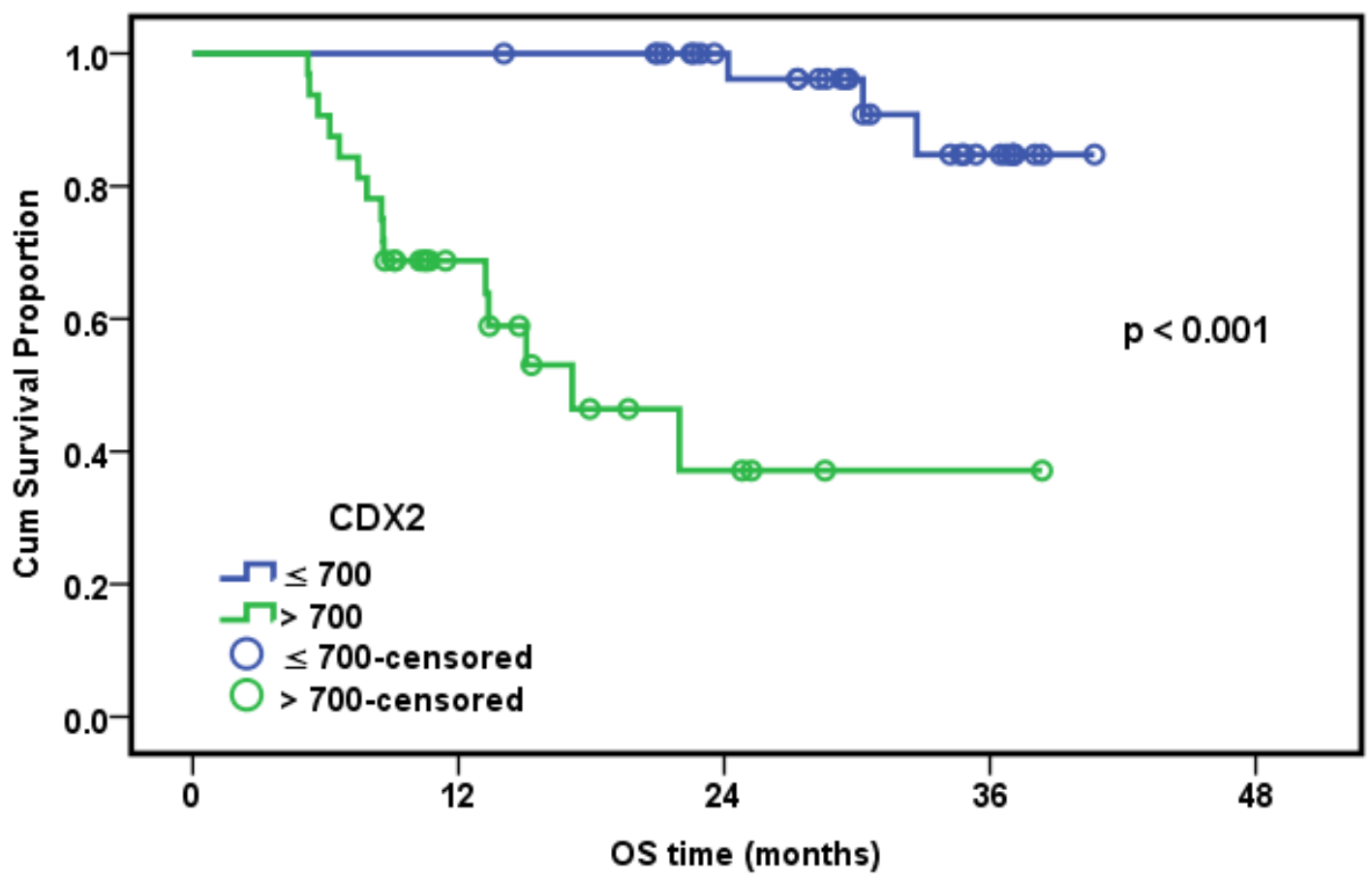

Fig 2:- Impact of $C D X 2$ Gene on Overall Survival (OS) of AML patients (High versus Low). 


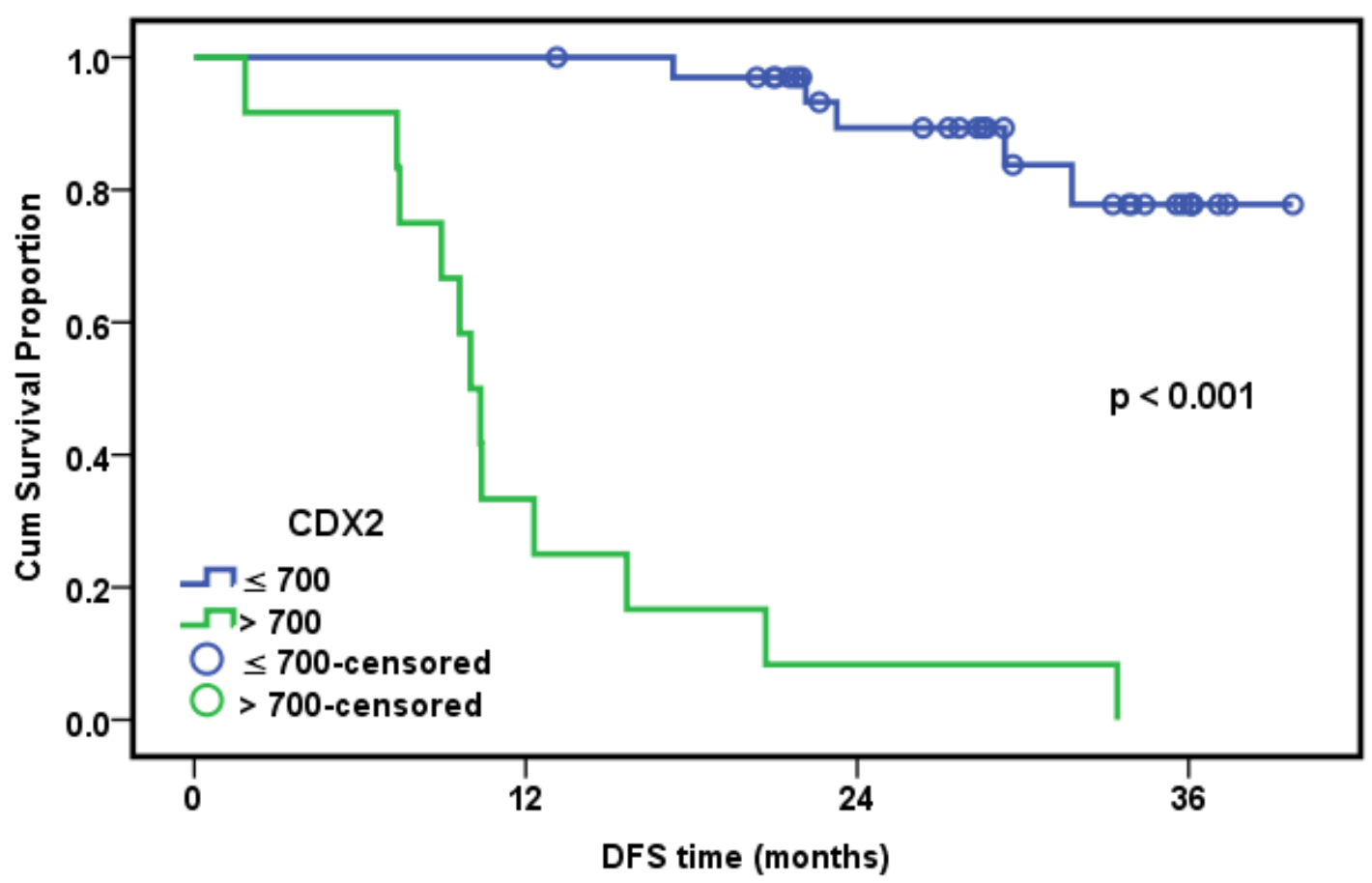

Fig 3:- Impact of CDX2 Gene on Disease Free Survival (DFS) of AML patients (High versus Low).

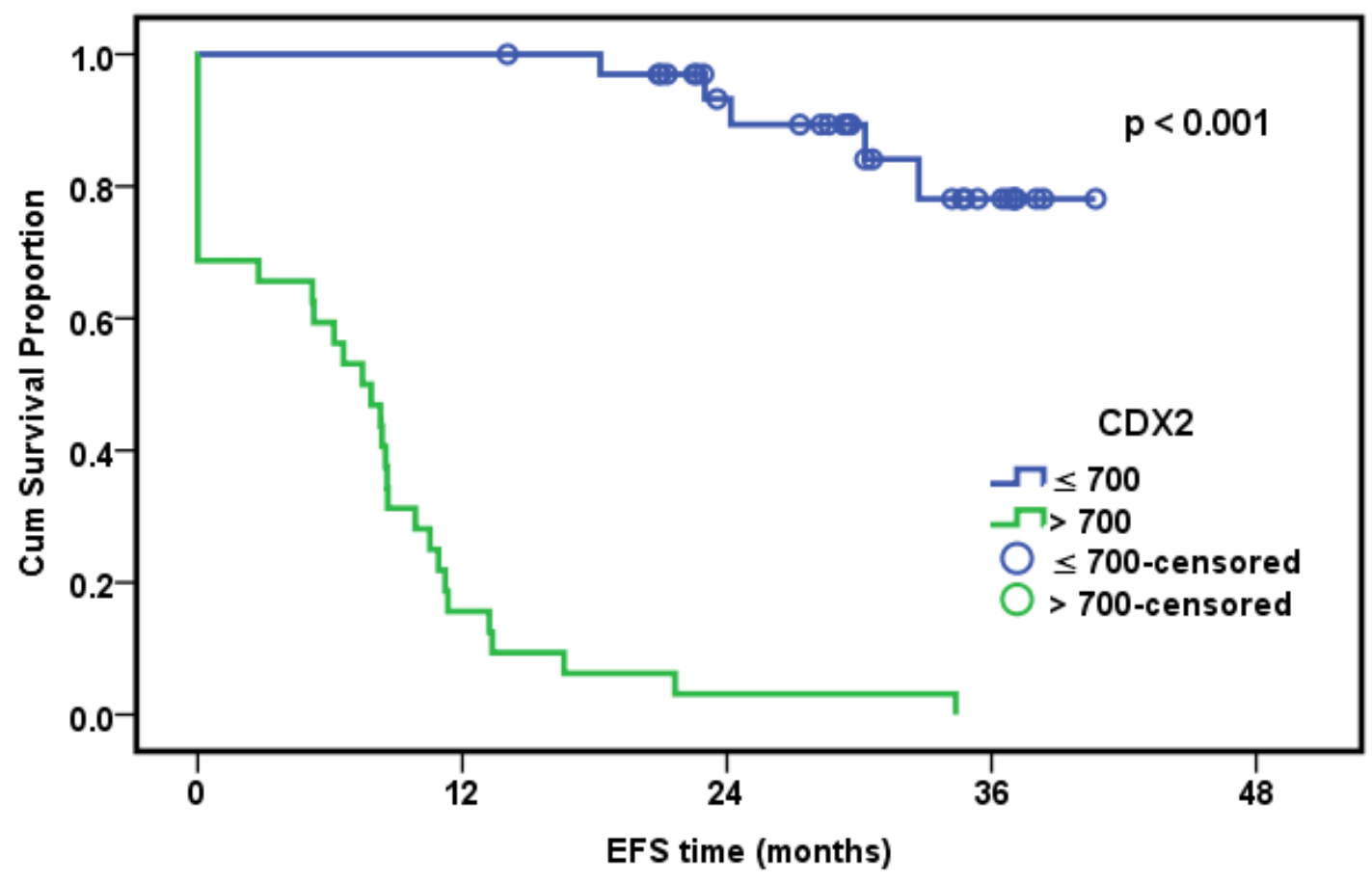

Fig 4:- Impact of CDX2 Gene on Event Free Survival (EFS) of AML patients (High versus Low).

\section{Discussion:-}

This study was performed in order to investigate the possible relationship between CDX2 gene expression and CNAML patients with a specific aim of determining gene relation to clinical features and laboratory findings at diagnosis, gene impact on patients' response to treatment in a case-control study including 66 de novo CN-AML patients and 20 healthy unrelated controls. 
Both the expression rates and expression levels of CDX2 mRNA were significantly higher in AML patients than normal controls $(\mathbf{p}<\mathbf{0 . 0 0 1})$. These findings were in agreement with previous studies ( $\mathbf{L i}$ et al., (2011), $\mathbf{L u}$ et al., (2012 and Wang et al., 2015). Rawat and colleagues in (2008) also found that CN-AML patients showed 14 folds higher expression levels of CDX2 compared to other AML patients.

The mechanism of aberrant expression of CDX2 in AML patients is not understood, but several theories tried to explain it. It could be explained by the known role of CDX2 as an upstream regulator of Hox genes whose up regulation has been associated with leukemogenesis and AML development. This support the hypothesis that CDX2 is responsible for the aberrant Hox gene expression that is observed in most AML cases and that CDX2 might play an important role in AML development with dysregulated Hox gene expression (Scholl et al., 2007 \& Rawat et al., 2008).

Another possible explanation is that CDX2 might deregulate multiple pathways in leukemia. CDX genes are upstream regulated by the retinoic acid (RA), the Wnt $/ \beta$ catenin and the fibroblast growth factor (FGF) signaling pathway which are dysregulated in human leukemias and might be responsible for this high CDX2 expression level in AML patients (Simon et al., (2005), Karajannis et al., (2006) \& Rawat et al., (2008). On the other hand, high CDX2 expression in leukemic and AML patients might result in activation of several pathways which are normally silent in early blood development (Rawat et al., 2004).

In-vitro experiments on BM transplanted mice model found that ectopic expression of CDX2 was highly leukemogenic and caused leukemia and AML in recipient mice (Rawat et al., 2004).

There was a wide range for CDX2 expression levels; so CDX2 expression was classified into high and low expressors according to its median expression level (700). Patients with higher CDX2 expression levels were older; had higher TLC and lower platelets count than CDX2 ${ }^{\text {low }}$ expressors. The difference was statistically significant $(\mathbf{p}=\mathbf{0 . 0 0 6})$ for age, $(\mathbf{p}=\mathbf{0 . 0 0 1})$ for TLC, $(\mathbf{p = 0 . 0 2 4})$ for platelets count. Also HSM and LN enlargements were more encountered in CDX2 high expressors. The difference was statistically significant $(\mathbf{p}<\mathbf{0 . 0 0 1 , 0 . 0 0 4})$ respectively. Patients with M0, M1 and M2 were more encountered in CDX2 ${ }^{\text {low }}$ expressors, while M4, M5 and M6 were more in CDX2 ${ }^{\text {high }}$ expressors. The difference was statistically significant $(\mathbf{p}=\mathbf{0 . 0 0 1})$.

In agreement with our findings, Lu et al., (2012) and Wang et al., (2015) also found that higher expression of CDX2 was significantly correlated with higher TLC. As far as we know, our study was the first study to correlate CDX2 in AML with age, platelets, organomegaly and FAB classifications. Previous studies on AML didn't report if there is a difference or not. Our findings could be explained by the higher the CDX2, the more the tumor burden and progression of disease.

When response of AML patients to therapy was considered; patients with high CDX2 expression were associated with worse prognosis than CDX2 ${ }^{\text {low }}$ expressors. All AML patients who didn't achieve CR were in CDX2 high expressors; CR patients were more in CDX2 ${ }^{\text {low }}$ than CDX2 ${ }^{\text {high }}$ expressors, the difference was highly statistically significant (p<0.001).

Our findings are in accordance with Lu et al., (2012) and Wang et al., (2015) who found that higher CDX2 expression levels were associated with lower CR rates. Wang et al., (2015) also reported that CDX2 expression level were decreased in AML patients after achieving CR but increased again in relapsing patients. Li et al., (2011), also found that CDX2 levels decreased in AL patients (ALL and AML) after achieving CR.

CDX2 ${ }^{\text {high }}$ expressors were associated with shorter OS, DFS and EFS than CDX2 ${ }^{\text {low }}$ expressors. The differences were highly statistically significant $(\mathbf{p}<\mathbf{0 . 0 0 1})$ in all.

As far as we know, this is the first study to correlate OS, DFS and EFS with CDX2 gene in AML patients, the previous studies in AML didn't correlate CDX2 with OS, DFS or EFS in AML patients. However, Riedt et al., (2009) found that higher CDX2 levels at diagnosis may correlate with more aggressive disease and with persistence of minimal residual disease (MRD) in pediatric ALL patients. They concluded that CDX2 expression levels may be used as a marker for bad prognosis in pediatric ALL. Thoene and colleagues (2009) also reported that higher CDX2 expression level was significantly associated with inferior OS in adult ALL patients. They also concluded that 
CDX2 is an independent risk factor in ALL patients. While Lu et al., (2011) found that ALL patients who achieved $\mathrm{CR}$ had lower CDX2 expression level which increased in relapsed patients.

In the current study, we found that CDX2 is a strong independent factor that influences OS, DFS and EFS when performing a multivariate study by Cox regression after adjusting the impact of other known risk factors.

\section{In Conclusion:-}

We found that CDX2 is highly expressed in CN-AML patients; its expression levels are associated with higher tumor burden. Higher CDX2 levels are associated with poor prognosis, lower CR rates and it is a strong independent factor affecting OS, DFS and EFS. Thus, CDX2 gene may be added to the prognostic markers in CN-AML patients.

\section{Conflict of interests:-}

The authors have no conflicts of interests to declare.

\section{Acknowledgements:-}

Thanks to Dr. Raafat Abdelfattah (Department of Clinical Oncology, National Cancer Institute, Cairo University) for helping us throughout this study.

\section{References:-}

- Abramovich C and Humphries R.K. (2005): Hox regulation of normal and leukemic hematopoietic stem cells. Curr. Opin. Hematol. 12:210-216.

- Argiropoulos B, Humphries RK. (2007): Hox genes in hematopoiesis and leukemogenesis. Oncogene.26 (47):6766-6776.

- Chawengsaksophak K, de Graaff W, Rossant, J, Deschamps J, and Beck F. (2004): Cdx2 is essential for axial elongation in mouse development. Proc. Natl. Acad. Sci. U. S. A. 101:7641-7645.

- Declaration of Helsinki, World Medical Association. (2008): Ethical Principles for Medical Research Involving Human Subjects, the 59th WMA General Assembly, Seoul, South Korea.

- Deneberg S, Guardiola P, Lennartsson A, Qu Y, Gaidzik V, Blanchet O, et al. (2011): Prognostic DNA methylation patterns in cytogenetically normal acute myeloid leukemia are predefined by stem cell chromatin marks. Blood. 118(20):5573-5582.

- Dohner H, Estey EH, Amadori S, Appelbaum FR, Buchner T, Burnett AK, et al. (2010): Diagnosis and management of acute myeloid leukemia in adults: recommendations from an international expert panel, on behalf of the European LeukemiaNet. Blood. 115(3):453-474.

- Dohner K, Schlenk RF, Habdank M, Scholl C, Rucker FG, Corbacioglu A, et al. (2005): Mutant nucleophosmin (NPM1) predicts favorable prognosis in younger adults with acute myeloid leukemia and normal cytogenetics: interaction with other gene mutations. Blood. 106:3740-3746.

- Garcia-Fernandez J. (2005): Hox, ParaHox, Proto-Hox: facts and guesses. Heredity. 94:145-152.

- Karajannis MA, Vincent L, Direnzo R, et al. (2006): Activation of FGFR1beta signaling pathway promotes survival, migration and resistance to chemotherapy in acute myeloid leukemia cells. Leukemia. 20:979-986.

- Kühnl A, Gökbuget N, Kaiser M, et al. (2011): Overexpression of LEF1 predicts unfavorable outcome in adult patients with B-precursor acute lymphoblastic leukemia. Blood. 8;118(24):6362-7.

- Li HY, Deng DH, Huang Y, Ye FH, Huang LL, Xiao Q, et al. (2015): Favorable prognosis of biallelic CEBPA gene mutations in acute myeloid leukemia patients: a meta-analysis. European journal of haematology. 94:439-48.

- Li YX, Pan KL, Fan F, Qian XH, Yang XL, Deng YL, Liu YD. (2011): Expression of cdx2 gene in pediatric patients with leukemia and its clinical significance. Zhongguo Shi Yan Xue Ye Xue Za Zhi. 19(1):130-3.

- Lu HY, Xia HL, Chen XW, Zhu LX, Wang QY, Cheng X. (2011): Expression and clinical significance of caudal type homeobox transcription factor-2 in adult acute lymphocytic leukemia. Zhongguo Shi Yan Xue Ye Xиe Za Zhi. 19(2):298-302.

- Lu Y, Wang Q, Mu QT, Yu MX, Chen ZM, Lou JY, Jin J. (2012): Expression level of CDX2 gene in acute myeloid leukemia and its clinical significance. Zhonghua Xue Ye Xue Za Zhi. 33(10):835-8.

- Lyu X, Xin Y, Mi R, Ding J, Wang X, Hu J, Fan R, Wei X, Song Y, Zhao RY. (2014): Overexpression of Wilms tumor 1 gene as a negative prognostic indicator in acute myeloid leukemia. PloS one. 9:e92470. 
- Mrozek K, Heerema NA, Bloomfield CD. (2004): Cytogenetics in acute leukemia. Blood reviews. 18(2):115136.

- Pineault N, Helgason CD, Lawrence HJ, Humphries RK. (2002): Differential expression of Hox, Meis1, and $\mathrm{Pbx} 1$ genes in primitive cells throughout murine hematopoietic ontogeny. Exp Hematol 2002; 30:49- 57.

- Rawat VP, Cusan M, Deshpande A, Hiddemann W, Quintanilla-Martinez L, Humphries RK, et al. (2004): Ectopic expression of the homeobox gene $\mathrm{Cdx} 2$ is the transforming event in a mouse model of t(12;13)(p13;q12)acute myeloid leukemia. Proc Natl Acad Sci U S A. 20;101(3):817-22.

- Rawat VP, Humphries RK, Buske C. (2012): Beyond Hox: the role of ParaHox genes in normal and malignant hematopoiesis. Blood. 19;120(3):519-27.

- Rawat VP, Thoene S, Naidu VM, Arseni N, Heilmeier B, Metzeler K, et al. (2008): Overexpression of CDX2 perturbs HOX gene expression in murine progenitors depending on its $\mathrm{N}$-terminal domain and is closely correlated with deregulated HOX gene expression in human acute myeloid leukemia. Blood. 1;111(1):309-19.

- $\quad$ Riedt T, Ebinger M, Salih HR, Tomiuk J, Handgretinger R, Kanz L, Grünebach F, Lengerke C. (2009): Aberrant expression of the homeobox gene CDX2 in pediatric acute lymphoblastic leukemia. Blood. 23;113(17):4049-51.

- Santos FP, Jones D, Qiao W, Cortes JE, Ravandi F, Estey EE, Verma D, Kantarjian H, Borthakur G. (2011): Prognostic value of FLT3 mutations among different cytogenetic subgroups in acute myeloid leukemia. Cancer. 117:2145-2155.

- Scholl C, Bansal D, Döhner K, Eiwen K, Huntly BJ, Lee BH, et al. (2007): The homeobox gene CDX2 is aberrantly expressed in most cases of acute myeloid leukemia and promotes leukemogenesis. J Clin Invest. 2007 Apr;117(4):1037-48.

- Schwind S, Marcucci G, Maharry K, Radmacher MD, Mrozek K, Holland KB, et al. (2010): BAALC and ERG expression levels are associated with outcome and distinct gene and microRNA expressionprofiles in older patients with de novo cytogenetically normal acute myeloid leukemia: a Cancer and Leukemia Group B study. Blood. 116:5660-5669.

- Shi JL, Fu L, Ang Q, Wang GJ, Zhu J, Wang WD. (2016): Overexpression of ATP1B1 predicts an adverse prognosis in cytogenetically normal acute myeloid leukemia. Oncotarget. 19;7(3):2585-95.

- Simon M, Grandage VL, Linch DC, Khwaja A. (2005): Constitutive activation of the Wnt/beta-catenin signalling pathway in acute myeloid leukaemia. Oncogene. 24:2410-2420.

- Tallman MS (2005): New strategies for the treatment of acute myeloid leukemia including antibodies and other novel agents. Hematology Am SocHematolEduc Program. 1:143-50.

- Thoene S, Rawat VP, Heilmeier B, et al. (2009): The homeobox gene CDX2 is aberrantly expressed and associated with an inferior prognosis in patients with acute lymphoblastic leukemia. Leukemia 23(4):649-655.

- Wang W, Meng C, Liu J, Yang Z. (2015): Expressions of CDX2 and $\beta$-catenin and their correlation in acute myeloid leukemia. Nan Fang Yi Ke Da Xue Xue Bao. 35(5):728-32. 